\title{
Um Framework para Apoio à Reflexão sobre o Processo de Produção de Objetos de Aprendizagem
}

\author{
João Pedro Dewes Guterres ${ }^{1}$ e Milene Selbach Silveira ${ }^{1}$ \\ ${ }^{1}$ Faculdade de Informática, Programa de Pós-Graduação em Ciência da Computação \\ Pontifícia Universidade Católica do Rio Grande do Sul (PUCRS) - Porto Alegre, RS - \\ Brasil \\ joao.guterres@acad.pucrs.br, milene.silveira@pucrs.br
}

\begin{abstract}
This paper presents a framework to support reflection on the process of learning object production. It is the result of the triangulation of related practices obtained from a literature review (systematic and mapping reviews), from observations and from interviews with members of nine Brazilian learning objects production centers. The format of the framework was based on a consolidated software engineering solution used by companies to qualify their software production process. The framework application was carried out in four production centers, demonstrating positive results for its capacity of self-reflection and incentive to carry out new practices. We hope the results could help both beginner and consolidated centers to qualify their processes through the self-reflection.
\end{abstract}

Resumo. Este artigo apresenta um framework para apoio à reflexão sobre o processo de produção de objetos de aprendizagem, resultado da triangulação de práticas pertinentes relacionadas ao tema obtidas a partir de uma revisão de literatura (incluindo uma revisão e um mapeamento sistemático), de observações e de entrevistas com integrantes de nove centros brasileiros de produção de objetos de aprendizagem. O formato do framework foi baseado em uma solução consolidada da Engenharia de Software, adotada por empresas para qualificação do processo de produção, e sua aplicação foi realizada em quatro centros de produção, demonstrando resultados positivos quanto a capacidade de autorreflexão e incentivo a realização de novas práticas. Espera-se que os resultados apresentados possam auxiliar tanto centros que estão iniciando esse processo quanto centros que já possuem suas práticas consolidadas, mas ainda assim possam qualificar seus processos.

\section{Introdução}

Segundo Carneiro e Silveira (2012), objetos de aprendizagem são "quaisquer materiais eletrônicos (como imagens, vídeos, páginas web, animações ou simulações), desde que tragam informações destinadas à construção do conhecimento (conteúdo autocontido), explicitem seus objetivos pedagógicos e estejam estruturados de tal forma que possam ser reutilizados e recombinados com outros objetos de aprendizagem (padronização)". Para apoiar o desenvolvimento desses recursos, diferentes processos de produção têm sido propostas [Braga et al. 2013; Kemczinski et al. 2012; Monteiro et al. 2006; Moraes et al. 2011]. Ao analisar esses processos, observa-se que, ao utilizarem, como base, abordagens próprias da produção de software, muitas adaptações necessitaram ser 
VI Congresso Brasileiro de Informática na Educação (CBIE 2017)

Anais do XXVIII Simpósio Brasileiro de Informática na Educação (SBIE 2017)

realizadas para comportar os aspectos pedagógicos inerentes a produção desses recursos educacionais.

Considerando a complexidade desta tarefa [Braga et al. 2013], inúmeras dificuldades são encontradas nesse processo, como o atraso na entrega devido ao retrabalho na produção [Santos 2009], a entrega de objetos sem as expectativas previstas [Romero et al. 2009], ou, mesmo, a produção de objetos não consistentes [Silveira e Carneiro 2012]. Assim, de modo a buscar uma solução para apoiar o processo de produção de objetos de aprendizagem, foram analisados trabalhos de pesquisa relacionados ao tema, iniciativas consolidadas na área de Engenharia de Software para qualificação da capacidade de desenvolvimento de software, como o CMMI e o MPS.BR [Kalinowski 2010], além da realização de entrevistas com participantes de centros brasileiros produtores de objetos de aprendizagem.

Este artigo apresenta o resultado desta pesquisa, o framework QPPOA, destinado a apoiar a reflexão sobre as práticas realizadas por centros de produção de objetos de aprendizagem, abordando a produção independente do tipo de objeto de aprendizagem desenvolvido, tamanho das equipes envolvidas, tecnologia adotada ou tipo de metodologia de produção utilizada. São apresentados, também, resultados da análise do framework com quatro centros de produção de objetos de aprendizagem.

O restante do artigo está organizado da seguinte forma: a seção 2 introduz a metodologia de pesquisa aplicada; na seção 3 é apresentado o framework QPPOA e a seção 4 a análise de sua aplicação; por fim, a seção 5 apresenta as considerações finais, além das limitações da pesquisa, contribuições e perspectivas de trabalhos futuros.

\section{Metodologia}

A produção de objetos de aprendizagem é uma tarefa de natureza interdisciplinar [Monteiro et al. 2006] envolvendo um trabalho colaborativo entre equipes multidisciplinares [Braga et al. 2013]. Primeiramente, buscou-se investigar processos de produção utilizados por pesquisadores brasileiros, sendo identificados 14 artigos citando 10 processos. Em busca de uma maior compreensão das práticas adotadas neste processo, foi realizada uma revisão de literatura mais abrangente incluindo uma revisão e um mapeamento sistemático. Na revisão sistemática, foram selecionadas 39 publicações internacionais, das quais foram extraídas informações sobre etapas, atividades, papéis, artefatos e sistemas envolvidos na produção de objetos. Já no mapeamento, foram selecionadas 205 publicações, das quais se buscou compreender o panorama brasileiro de produção de objetos, incluindo práticas e metodologias adotadas nesse processo.

De modo a capturar uma perspectiva prática do processo de produção, buscou-se por centros de produção de objetos de aprendizagem. Foram identificados 17 centros ativos e foi possível realizar as entrevistas com 9 deles. $O$ escopo dos centros de produção entrevistados englobou características abrangentes, sendo a produção nestes centros diversificada entre tipos de objetos produzidos, número de papéis envolvidos e tipos de metodologias adotadas na produção. E o conjunto de entrevistados incluiu 8 coordenadores, 4 desenvolvedores, 1 designer instrucional e 1 analista.

Por meio de uma entrevista semiestruturada, buscou-se abranger todo o processo de produção, incluindo a experiência do entrevistado, o processo de produção seguido, 
VI Congresso Brasileiro de Informática na Educação (CBIE 2017)

Anais do XXVIII Simpósio Brasileiro de Informática na Educação (SBIE 2017)

as formas de registro de atividades e os papéis envolvidos. Para a análise dos dados, optou-se por utilizar a metodologia de pesquisa Grounded Theory [Corbin e Strauss 2008], adotando as entrevistas como fonte primária de dados e as publicações e observações do pesquisador como fontes secundárias para triangulação de dados.

A partir da aplicação desta metodologia sobre os dados, foram realizadas as codificações aberta, axial e seletiva dos dados, dando origem a categorias, hipóteses e inter-relações entre elas. Com base nessa análise, foram elaboradas teorias sobre as práticas realizadas na produção de objetos de aprendizagem. Essas teorias foram segmentadas em duas dimensões de análise: práticas específicas por etapa de produção e práticas aplicáveis ao processo de produção como um todo. A compilação dessas teorias deu origem ao framework proposto, o qual contém 22 práticas.

O processo de aplicação do framework em centros de produção envolveu a auto avaliação dos centros referente a aderência das práticas por eles realizadas, contrastadas com as práticas previstas no framework. Além disso, houve uma entrevista semiestruturada discutindo a aplicação. Ao todo, 4 centros de produção fizeram a aplicação, em um total de 5 representantes (em um centro houve a aplicação por 2 representantes). A primeira aplicação - análise piloto - foi realizada presencialmente e as demais de modo remoto: o framework foi enviado por e-mail e, após sua aplicação, foi realizada a entrevista.

\section{Framework QPPOA}

Esta seção descreve o framework concebido para a apoiar a reflexão sobre as práticas realizadas durante o processo de produção de objetos de aprendizagem. Elas foram segmentadas entre as cinco etapas do processo, além de um conjunto que é aplicável ao processo como um todo (Figura 1).

Cada prática do framework contém a descrição de seu propósito, atividades envolvidas, além de sugestões e comentários sobre como aplicá-la. A seguir elas estão apresentadas de forma resumida, com a descrição de cada prática e as atividades relacionadas, de acordo com a respectiva etapa ${ }^{1}$.

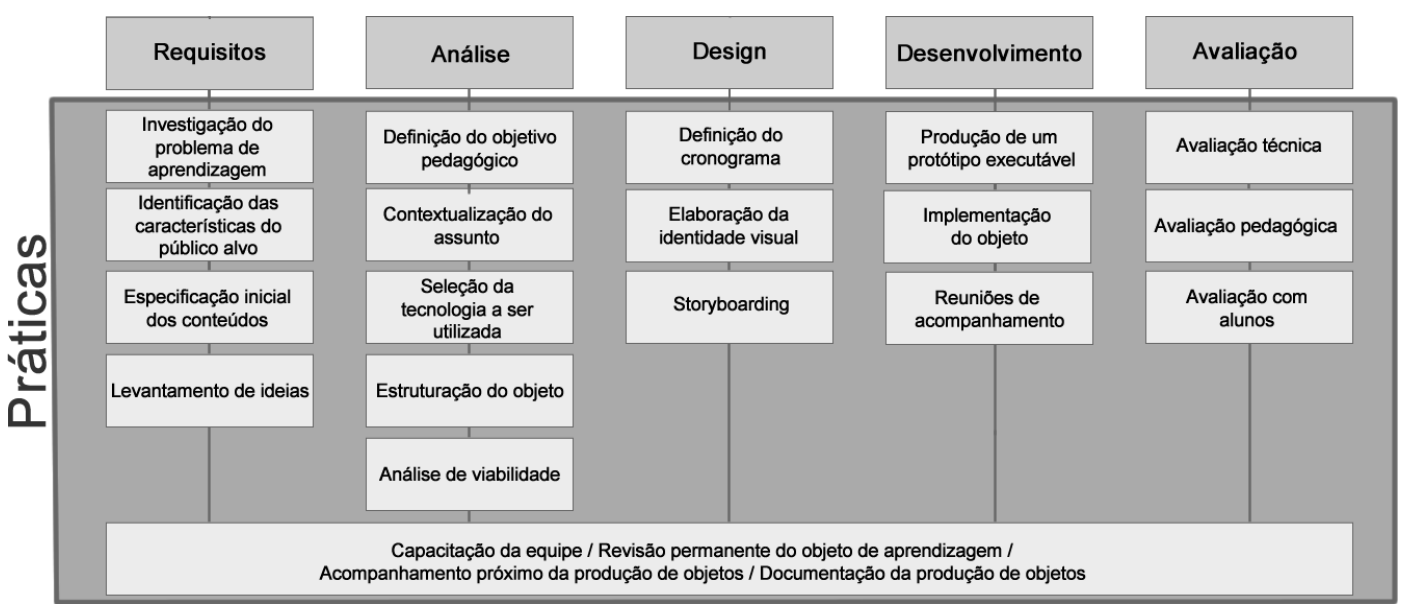

Figura 1. Práticas do framework.

\footnotetext{
${ }^{1} \mathrm{O}$ detalhamento completo das práticas e seu protocolo de aplicação pode ser acessado em https://drive.google.com/file/d/0B7H8lbJM9Nj5RVQ5Z2VjWXVRU1E/view?usp=sharing.
} 
VI Congresso Brasileiro de Informática na Educação (CBIE 2017)

Anais do XXVIII Simpósio Brasileiro de Informática na Educação (SBIE 2017)

\subsection{Etapa de Requisitos}

O objetivo desta etapa é realizar um levantamento sobre o público alvo e dificuldades de aprendizagem que guiem a especificação inicial, além de ideias para enfrentar tais obstáculos (Tabela 1).

Tabela 1. Práticas da etapa de Requisitos e suas respectivas atividades.

\begin{tabular}{|l|l|}
\hline $\begin{array}{l}\text { Investigação do } \\
\text { Problema de } \\
\text { Aprendizagem }\end{array}$ & $\begin{array}{l}\text { Identificação, por parte dos professores, de dificuldades de } \\
\text { aprendizagem em determinados conteúdos; Percepção, por parte } \\
\text { do professor ou equipe pedagógica, que uma abordagem interativa } \\
\text { relacionada a determinado conteúdo melhoraria a compreensão do } \\
\text { aluno, superando determinada dificuldade. }\end{array}$ \\
\hline $\begin{array}{l}\text { Identificação das } \\
\text { Características do } \\
\text { Público Alvo }\end{array}$ & $\begin{array}{l}\text { Elaboração do perfil do aluno (idade, gênero, preferências, etc.); } \\
\text { Consideração da acessibilidade dos recursos a estes alunos, sendo } \\
\text { o recurso disponível em qualquer local e para qualquer pessoa. }\end{array}$ \\
\hline $\begin{array}{l}\text { Especificação } \\
\text { Inicial dos } \\
\text { Conteúdos }\end{array}$ & $\begin{array}{l}\text { Organização de materiais e informações relacionadas, como pré- } \\
\text { requisitos, sobre o conteúdo do problema identificado; } \\
\text { Apresentação, por parte dos professores, dos materiais à equipe } \\
\text { pedagógica e técnica sobre o tema abordado. }\end{array}$ \\
\hline $\begin{array}{l}\text { Levantamento de } \\
\text { Ideias }\end{array}$ & $\begin{array}{l}\text { Apresentação, pela equipe técnica de ideias sobre recursos } \\
\text { tecnológicos, e apresentação das limitações técnicas das ideias, } \\
\text { incluindo narrativas, navegação, design e tecnologias; Debate de } \\
\text { ideias sobre como apresentar o conteúdo em forma de objeto. }\end{array}$ \\
\hline
\end{tabular}

\subsection{Etapa de Análise}

O objetivo desta etapa é investigar os requisitos elaborados, resultando num esboço inicial do objeto com seu conteúdo contextualizado, estruturado, além da escolha da tecnologia adotada com sua viabilidade de produção (Tabela 2).

Tabela 2. Práticas da etapa de Análise e suas respectivas atividades.

\begin{tabular}{|l|l|}
\hline $\begin{array}{l}\text { Definição do } \\
\text { Objetivo } \\
\text { Pedagógico }\end{array}$ & $\begin{array}{l}\text { Justificativa do motivo da construção do objeto de aprendizagem; } \\
\text { Descrição do objetivo pedagógico; Descrição do público alvo e } \\
\text { quais dificuldades o objeto busca solucionar. }\end{array}$ \\
\hline $\begin{array}{l}\text { Contextualização } \\
\text { do Assunto }\end{array}$ & $\begin{array}{l}\text { Criação de situações-problema com base nos problemas } \\
\text { identificados; Contextualização das situações com um desafio, } \\
\text { estudo de caso, etc.; Escolha de personagens e elementos lúdicos. }\end{array}$ \\
\hline $\begin{array}{l}\text { Seleção da } \\
\text { Tecnologia } \\
\text { Utilizada }\end{array}$ & $\begin{array}{l}\text { Apresentação de modelos pré-existentes de objetos, de modo a } \\
\text { exibir as possibilidades dos recursos; Exposição das limitações e } \\
\text { possibilidades das tecnologias selecionadas. }\end{array}$ \\
\hline $\begin{array}{l}\text { Estruturação do } \\
\text { Objeto }\end{array}$ & $\begin{array}{l}\text { Definição dos principais elementos do objeto, tais como } \\
\text { personagens, animações, vídeos, etc; Estruturação e decomposição } \\
\text { dos principais conteúdos do objeto de aprendizagem. }\end{array}$ \\
\hline $\begin{array}{l}\text { Análise de } \\
\text { Viabilidade }\end{array}$ & $\begin{array}{l}\text { Avaliação de viabilidade, pela equipe técnica, das atividades } \\
\text { propostas no estruturação inicial do objeto; Definição do reuso de } \\
\text { elementos no objeto. }\end{array}$ \\
\hline
\end{tabular}


VI Congresso Brasileiro de Informática na Educação (CBIE 2017)

Anais do XXVIII Simpósio Brasileiro de Informática na Educação (SBIE 2017)

\subsection{Etapa de Design}

O objetivo desta etapa é a elaboração do roteiro final (storyboarding) do objeto, juntamente com sua identidade visual e cronograma das tarefas (Tabela 3 ).

Tabela 3. Práticas da etapa de Design e suas respectivas atividades.

\begin{tabular}{|l|l|}
\hline $\begin{array}{l}\text { Definição do } \\
\text { Cronograma }\end{array}$ & $\begin{array}{l}\text { Identificação de tarefas concorrentes e dependentes; Definição } \\
\text { dos papéis responsáveis pelas tarefas; Estipulação de prazos e } \\
\text { encadeamento das tarefas. }\end{array}$ \\
\hline $\begin{array}{l}\text { Elaboração da } \\
\text { Identidade Visual }\end{array}$ & $\begin{array}{l}\text { Elaboração dos principais elementos de design; Prototipação de } \\
\text { baixa fidelidade para validação; Validação da identidade visual. }\end{array}$ \\
\hline Storyboarding & $\begin{array}{l}\text { Estruturação dos conteúdos; Orientações sobre eventos dinâmicos } \\
\text { dos conteúdos e animações. }\end{array}$ \\
\hline
\end{tabular}

\subsection{Etapa de Desenvolvimento}

O objetivo desta etapa é a prototipação e implementação do objeto, incluindo revisões cíclicas e reuniões de acompanhamento (Tabela 4).

Tabela 4. Práticas da etapa de Desenvolvimento e suas respectivas atividades.

\begin{tabular}{|l|l|}
\hline $\begin{array}{l}\text { Produção de um } \\
\text { Protótipo Executável }\end{array}$ & $\begin{array}{l}\text { Implementação dos principais elementos do objeto; } \\
\text { Validação do protótipo. }\end{array}$ \\
\hline $\begin{array}{l}\text { Implementação do } \\
\text { Objeto }\end{array}$ & $\begin{array}{l}\text { Implementação incremental do objeto; Revisões cíclicas de } \\
\text { qualidade. }\end{array}$ \\
\hline Reuniões de & $\begin{array}{l}\text { Exposição do progresso, por parte dos desenvolvedores, das } \\
\text { Acompanhamento }\end{array}$ \\
$\begin{array}{l}\text { suas tarefas; Análise das dificuldades e realização de ajustes, } \\
\text { por parte da coordenação, na distribuição de tarefas; } \\
\text { Documentação do progresso das tarefas e dificuldades } \\
\text { encontradas. }\end{array}$ \\
\hline
\end{tabular}

\subsection{Etapa de Avaliação}

O objetivo desta etapa é verificar se o objeto produzido atende às especificações em aspectos técnicos e pedagógicos, incluindo uma avaliação piloto com alunos, de modo a validar a eficácia do objeto em auxiliar o processo de aprendizagem (Tabela 5).

Tabela 5. Práticas da etapa de Avaliação e suas respectivas atividades.

\begin{tabular}{|l|l|}
\hline Avaliação Técnica & $\begin{array}{l}\text { Testes de unidade na implementação dos incrementos; Testes } \\
\text { de integração na integração dos incrementos. }\end{array}$ \\
\hline Avaliação Pedagógica & $\begin{array}{l}\text { Avaliação de adequação do objeto a seu objetivo pedagógico; } \\
\text { Verificação da adequação pedagógica do objeto de } \\
\text { aprendizagem às características do público alvo. }\end{array}$ \\
\hline $\begin{array}{l}\text { Avaliação Piloto com } \\
\text { Alunos }\end{array}$ & $\begin{array}{l}\text { Coleta de dados sobre o uso do objeto pelos alunos; Análise } \\
\text { das dificuldades encontradas no uso do objeto; Avaliação da } \\
\text { eficácia do objeto; Documentação da avaliação com alunos. }\end{array}$ \\
\hline
\end{tabular}

\subsection{Práticas Aplicáveis ao Processo como um Todo}

Estas práticas estão relacionadas a aspectos do centro de produção não atrelados a nenhuma etapa específica, procedendo como princípios envolvidos na produção de objetos (Tabela 6). 
VI Congresso Brasileiro de Informática na Educação (CBIE 2017)

Anais do XXVIII Simpósio Brasileiro de Informática na Educação (SBIE 2017)

Tabela 6. Outras práticas e suas respectivas atividades.

\begin{tabular}{|l|l|}
\hline $\begin{array}{l}\text { Capacitação da } \\
\text { Equipe }\end{array}$ & $\begin{array}{l}\text { Capacitação da equipe técnica sobre os conceitos envolvidos na } \\
\text { elaboração dos objetos; Capacitação da equipe pedagógica } \\
\text { sobre quais recursos são possíveis de se implementar. }\end{array}$ \\
\hline $\begin{array}{l}\text { Revisão Permanente } \\
\text { dos Objetos de } \\
\text { Aprendizagem }\end{array}$ & $\begin{array}{l}\text { Verificação dos conteúdos após uma determinada data; } \\
\text { Atualização de tecnologia, se verificada falta de suporte; } \\
\text { Refinamento na programação, tornando objetos mais modulares } \\
\text { e facilitando o reuso. }\end{array}$ \\
\hline $\begin{array}{l}\text { Acompanhamento } \\
\text { Próximo da }\end{array}$ & $\begin{array}{l}\text { Utilização de software de gerenciamento e alocação de } \\
\text { atividades; Realocação de equipe para atender as demandas } \\
\text { mais urgentes; Gerencia de conflitos de atividades e demandas. }\end{array}$ \\
\hline $\begin{array}{l}\text { Documentação da } \\
\text { Produção de objetos }\end{array}$ & $\begin{array}{l}\text { Documentação de atas de reuniões; Documentação das ideias e } \\
\text { decisões tomadas; Documentação das dificuldades e soluções } \\
\text { encontradas. }\end{array}$ \\
\hline
\end{tabular}

\section{Análise do Framework}

A utilização do framework em centros de produção de objetos visou analisar sua aplicabilidade e pontos de melhoria, considerando-se, para isto, que o framework deveria ser: compreensível e autocontido aos participantes da avaliação; ter facilidade de aplicação; e possibilitar uma classificação clara de aderência das práticas realizadas

A aplicação do framework foi realizada em 4 dos 9 centros de aprendizagem entrevistados na primeira etapa, sendo estes centros experientes na produção de objetos com mais de 6 anos de produção. O framework foi aplicado por 5 representantes de 4 centros de produção de objetos de aprendizagem (como um dos centros possui a coordenação dividida entre coordenação pedagógica e técnica, optou-se por incluir ambas). Para identificação dos representantes dos centros em suas avaliações, adotamos as identificações C1, C2, C3 e C4.1 e C4.2 (sendo C4 o centro com 2s representantes).

Para avaliação de aderência das práticas, foi adotada uma adaptação da classificação SPICE [ISO/IEC 15504 2003]: Não Atingido, pouca ou nenhuma evidência de realização ( 0 a $5 \%$ de evidência); Parcialmente Atingido, alguma evidência e realizações relativas (de 15 a 50\%); Largamente Atingido, evidências de uma realização significativa, porém com algumas fraquezas (de 50 a 85\%); e Totalmente Atingido, evidências de uma abordagem sistemática (de 85 a 100\%).

Analisando as classificações dos centros sobre suas práticas, observa-se que das 22 práticas, apenas três delas ("Levantamento de ideias", "Definição do objetivo pedagógico" e "Seleção da tecnologia utilizada") foram classificadas como totalmente atingidas por todos os centros. E, analisando as classificações somadas de todos os representantes dos centros, somam-se 13 de suas práticas como não atingidas, 16 como parcialmente atingidas, 39 como largamente atingidas e 42 como totalmente atingidas. Considerando estes resultados, evidencia-se a larga experiência destes centros, que já possuem práticas consolidadas no processo de produção.

Especificamente analisando os resultados das práticas da etapa de Requisitos, a autoanálise dos centros sobre suas práticas gerou resultados pouco dispersos, a maioria estando entre largamente e totalmente atingido. Os representantes enfocaram a importância das práticas adotadas, entretanto, que algumas delas são realizadas 
VI Congresso Brasileiro de Informática na Educação (CBIE 2017)

Anais do XXVIII Simpósio Brasileiro de Informática na Educação (SBIE 2017)

parcialmente pelo centro de produção, sendo os professores (autores dos objetos) - que muitas vezes são externos a equipe de produção - os protagonistas dessas atividades. C4.2 comenta esse fato: "Trabalhamos com as demandas dos professores, assessoramos o seu trabalho de construção de conteúdos e objetos".

Já os resultados da etapa de Análise foram mais expressivos, sendo a grande maioria das práticas avaliadas como totalmente atingida. Nesse panorama, o professor como protagonista é mencionado por C3: "são colaborações pequenas, do ponto de vista pedagógico, em comparação com o trabalho feito pelo professor". Já C4.2 cita a presença da orientação por membros específicos da equipe de produção para esta tarefa: “Todo esse processo é orientado e acompanhado pelos orientadores pedagógicos".

$\mathrm{Na}$ etapa de Design, as avaliações foram centralizadas em largamente atingidas, indicando a existência de pontos de melhorias para atingirem a totalidade de aderência sobre as práticas. Nesse sentido, $\mathrm{C} 1$ cita a falta de programas específicos para gerenciar as atividades realizadas: "eu sinto falta é o uso de uma ferramenta de gerenciamento (...) eu noto que fica muito a cargo do gerente, (...), na ausência dele fica complicado". $\mathrm{Na}$ prática de Elaboração da identidade visual, C4.1 relatou a realização da prática, porém, não sendo aplicada de forma sistemática a todos os objetos: "A identidade visual é sempre adaptada à demanda específica. Marquei como largamente atingido porque nem sempre realizamos prototipação ou uso de wireframes".

A etapa de Desenvolvimento foi também classificada como largamente atingida, indicando, a ausência de uma sistematização das práticas. C4.1 comenta essa característica: "Falta uma sistematização das revisões cíclicas de qualidade". Já na prototipação, $\mathrm{C} 1$ relata que mesmo com objeto bem encaminhado, a prototipação é algo importante: "é tanta discussão nessa fase inicial (...) que quando ele chega nessa fase ele já tá pronto praticamente. O que não é o correto de fazer não, porque o correto é ainda nós fazermos um executável". Como autorreflexão de melhoria na atividade de documentação, C2 relata: "essa documentação é no final e ela poderia ser feita gradativamente.".

$\mathrm{Na}$ etapa de Avaliação houve uma dispersão maior entre as classificações, principalmente pela falta de tempo para realização de testes. Nesse cenário, C3 justificou que isto é devido a pouca complexidade dos objetos, não havendo necessidade de avaliações técnicas, e as pedagógicas só são realizadas quando há interesse de pesquisa com os objetos. Já $\mathrm{C} 1$ menciona que só realiza testes quando ocorrem erros: "isso só é verificado se por ventura aparecer algum problema, se não aparecer não é verificado". Sobre a prática de avaliação com alunos, C4.1 comenta a classificação de não atingido: "Embora já tenham ocorrido avaliações com alunos, esta prática não é frequente e não é requisito para publicação". Por outro lado, C1 e C2 relatam a importância dessa atividade para finalizar a produção dos objetos, considerando a aprendizagem do aluno e se sua estrutura está adequada.

Por fim, as práticas que se aplicam ao processo como um todo foram classificadas, em sua maioria, como não atingidas e parcialmente atingidas. Sobre as capacitações, é mencionado que a frequência delas está diminuindo, principalmente por serem já experientes em seus processos. Nesse cenário, C2 relata algumas dificuldades, principalmente por conta da participação de novos professores não capacitados: "por mais que a gente tenha a equipe preparada, mas não é fácil convencer o docente, 
VI Congresso Brasileiro de Informática na Educação (CBIE 2017)

Anais do XXVIII Simpósio Brasileiro de Informática na Educação (SBIE 2017)

porque é um docente que já vem com uma visão sobre EaD ou objetos, como eles tem que ser e é um pouco dificil ter que reconstruir isso e nem sempre a gente consegue".

Sobre a experiência dos centros na avaliação das práticas por algum outro tipo de framework, ela foi inédita para todos os centros entrevistados, tanto para experiência com frameworks da Engenharia de Software quanto da produção de objetos. O representante $\mathrm{C} 4.2$ relatou o desconhecimento do uso de frameworks para qualificar o processo de produção: "eu não conhecia. Foi a primeira vez que nós pegamos, principalmente tão voltado para a parte pedagógica que foi bastante interessante.".

Questionados sobre a capacidade do framework em qualificar o processo de produção, $\mathrm{C} 1$ afirmou, mencionando a abrangência do framework: "sim, até porque concilia nesse framework a importância da Engenharia de Software e IHC, isso é importante para o processo de desenvolvimento e você contemplou muito bem isso, essa relação. Por muito tempo ela foi dissociada, mas não dá mais para fazer vistas grossas não, elas são juntas sim.". Já C4.2 ressalta que o framework sozinho não é uma solução completa para a produção de objetos, mas que também, pode ser utilizado como um guia para montar e avaliar o processo de produção, servindo como ponto de autorreflexão: "enquanto eu respondia o teu framework eu ficava me perguntando se nós realmente estávamos fazendo como deveria, e fiquei pensando o quanto a gente devia mudar ou melhorar ou aperfeiçoar as nossas práticas então eu acho que ele realmente serve nesse sentido.".

C4.1 comenta a abrangência do framework: "achei bem interessante, é um framework que cria um caminho bem claro, bem objetivo e certamente vai inclusive nos ajudar aqui a rever o nosso processo e eu percebi que a gente faz praticamente tudo, só que claro que nem sempre é $100 \%$ (...) serve sim, tanto para instituições de pequena, média e grande porte". Já C3 mencionou não saber se qualifica o processo, apesar de reconhecer a importância de algumas práticas avaliadas: "não sei, vendo as coisas que tem todas elas são extremamente importante, (...) não posso fazer um objeto em função de cronograma, não dá certo, mas estrutura do objeto, análise de viabilidade, especificação, isso é uma coisa que não dá para fazer se não tiver (...) só que do jeito que a gente aprendeu a fazer aqui com o passar do tempo é por tentativa e erro por experiência.".

Sendo a produção de objetos de aprendizagem uma tarefa complexa, que é realizada de diferentes formas, com diferentes tipos de demanda e equipes, houve alguns pontos de melhoria observados pelos representantes para que o framework possa ser mais abrangente a todos os centros. Essa evidência foi comentada principalmente na necessidade de tratar melhor algumas características, como as diferentes formas de demanda e produção e interações entre os papeis envolvidos em cada prática. Cita-se, nesse contexto, principalmente o papel do professor como ora papel interno ao centro de produção ora papel externo, apenas demandando a produção do objeto.

Sobre a realização das práticas, um dos fatores constantemente levantado pelos representantes dos centros foi o pouco de tempo para realizar algumas práticas, relacionando esse fator à necessidade de uma produção sustentável e rápida para dar conta da alta demanda. Para chegar nessa produção, os relatos indicam que os centros realizaram muitas experiências, por tentativa e erro muitas vezes, para chegar num processo que realize somente as práticas essenciais à produção e que dê resultados satisfatórios, considerando tempo, escopo e qualidade dos objetos de aprendizagem. 
Contrastando a realidade vivida pelo centro e as práticas analisadas, apenas a prática "Avaliação com alunos" recebeu críticas por ser considerada inviável pelos representantes do centro $\mathrm{C} 4$. Esse relato vem ao encontro dessa produção sustentável relatada por C4.1. Esse indício também se relaciona a característica de produção seguida pelo centro, sem um público alvo por ser multinível para diversos públicos. Nesse contexto, assim como foi relatado pelo $\mathrm{C} 4$, o framework visa formular práticas ideais, mesmo que inviáveis a determinados centros, visto que estas proporcionam benefícios ao integrar a percepção do aluno sobre o objeto antes da finalização. Já C3, mencionou que o framework foge um pouco da realidade vivida por conta dos tipos de objetos produzidos e o modo de implementação, modo individual pelos desenvolvedores. Nesse sentido, o framework talvez não seja adequado para avaliar o processo de produção de todos os materiais produzidos, destacando a grande variedade de recursos que podem ser denominados objetos de aprendizagem.

De modo geral, o framework foi capaz de propiciar, aos representantes dos centros, uma visão geral do processo de produção, subdividindo-o em práticas que os auxiliam na compreensão de grande parte das atividades relevantes que costumam ser realizadas durante esse processo. E foi útil, principalmente, como um material de análise (e autorreflexão) de cada centro sobre suas próprias atividades.

\section{Conclusões}

Essa pesquisa apresenta um framework para apoiar a autorreflexão de centros de produção de objetos de aprendizagem sobre as práticas por eles realizadas. Para concepção do framework, foram realizados estudos teóricos e práticos que buscaram compreender este processo como um todo.

A aplicação do framework trouxe um maior conhecimento das práticas realizadas nos centros de produção de objetos de aprendizagem. A autorreflexão dos centros a partir da avaliação das práticas, citada como uma importante ferramenta capaz de apoiar a qualificação do processo de produção, foi percebida na maioria dos centros, de forma indireta, pela indicação de realização de novas práticas, e direta, sendo citada, por exemplo, a necessidade de rever o processo de produção após o uso do framework. Ressalta-se que o framework foi elaborado para auxiliar os centros de produção, independente de sua metodologia, tamanho de equipe ou tecnologia adotada na implementação dos objetos.

Como limitação da pesquisa, cita-se o escopo da fonte primária de dados e os centros que realizaram a avaliação do framework, sendo estes centros universitários brasileiros de produção de objetos com experiência relativamente grande neste processo. Nesse panorama, a ausência de informações e avaliações com centros de produção iniciantes (menor porte), pode ausentar aspectos importantes da produção.

Como próximos passos da pesquisa, espera-se também realizar a aplicação do framework em centros com processos menos consolidados, além de retornar aos centros entrevistados, depois de um período de 6 meses, para discutir o impacto da autorreflexão. Além disso, cita-se a necessidade de um aprofundamento do framework com novas práticas, além de revisões sobre as práticas já elencadas, indicando, por exemplo, quais são os benefícios imediatos e em longo prazo de sua aplicação. 
VI Congresso Brasileiro de Informática na Educação (CBIE 2017)

Anais do XXVIII Simpósio Brasileiro de Informática na Educação (SBIE 2017)

Espera-se que os resultados apresentados possam auxiliar tanto centros que estão iniciando o processo de produção de objetos de aprendizagem quanto centros que já possuem suas práticas consolidadas e, por meio de autorreflexão destas práticas, possam qualificar seus processos.

\section{Agradecimentos}

João Pedro Guterres agradece à PROSUC/CAPES pela concessão de bolsa de doutorado. O presente trabalho foi realizado com apoio do $\mathrm{CNPq}$, Conselho Nacional de Desenvolvimento Científico e Tecnológico Brasil por concessão de bolsa de mestrado.

\section{Referências}

Braga, J.C.; Pimentel, E.; Dotta, S. (2013) "Metodologia INTERA para o desenvolvimento de Objetos de Aprendizagem". In: Simpósio Brasileiro de Informática na Educação, vol. 24, p. 206-315.

Carneiro, M.L.F.; Silveira, M.S. (2012) "Objetos de aprendizagem sob o ponto de vista dos alunos: um estudo de caso”. In RENOTE, vol. 10(3), 10p.

Corbin, J.; Strauss, A. "Basics of qualitative research: Techniques and procedures for developing grounded theory”. California: Sage Publications, vol. 3, 2008.

ISO/IEC 15504 - Part 1: Concepts and Vocabulary (2003).

Kalinowski, M.; Santos, G.; Reinehr, S.; Montoni, M. Rocha, A.R.; Weber, K.C.; Travassos, G.H. (2010) "MPS. BR: promovendo a adoção de boas práticas de engenharia de software pela indústria brasileira." In: Congreso Iberoamericano en Software Engineering, vol. 13, 14p.

Kemczinski, A.; Costa, I.A.; Wehrmeister, M.A.; Hounsell, M.S.; Vahldick, A. (2012) "Metodologia para Construção de Objetos de Aprendizagem Interativos". In: Simpósio Brasileiro de Informática na Educação, vol. 23, 10p.

Monteiro, B.D.S.; Cruz, H. P.; Andrade, M.; Gouveia, T.; Tavares, R.; dos Anjos, L. F. (2006) "Metodologia de desenvolvimento de objetos de aprendizagem com foco na aprendizagem significativa". In: Simpósio Brasileiro de Informática na Educação, vol. 17, p. 388-397.

Moraes, M.; Raymundo, V.; Bocchese, J.; Lima, V. (2011) "Elaboração de Objetos de Aprendizagem para o LAPREN: Processo de Desenvolvimento e Sistema de Produção”. In: Simpósio Brasileiro de Informática na Educação, vol. 22, p. 224-233.

Romero, T.R.L.; Andrade, R.; Pietrocola, M. (2009) "Parâmetros para análise de roteiros de objetos de aprendizagem". In: Simpósio Nacional de Ensino de Física, vol. $18,11 \mathrm{p}$.

Santos, N.S.R.S. (2009) "Processo produtivo de objetos de aprendizagem: problemas e soluções". Dissertação de Mestrado em Engenharia de Produção, UENF, 147p.

Silveira, M.S.; Carneiro, M.L.F. (2012) "Desconstruindo Objetos de Aprendizagem: reflexões sobre sua qualidade de uso". In: Simpósio Brasileiro de Informática na Educação, vol. 23, 10p. 